\section{Vitamin D bei Schwangeren im Auge behalten}

Wissenschaftler aus den Niederlanden konnten belegen, dass ein

Vitamin-D-Mangel bei Schwangeren keine Seltenheit ist. Dieser erhöht die Empfänglichkeit des Säuglings für Infektionen mit dem respiratorischen Synzytialvirus (RSV).

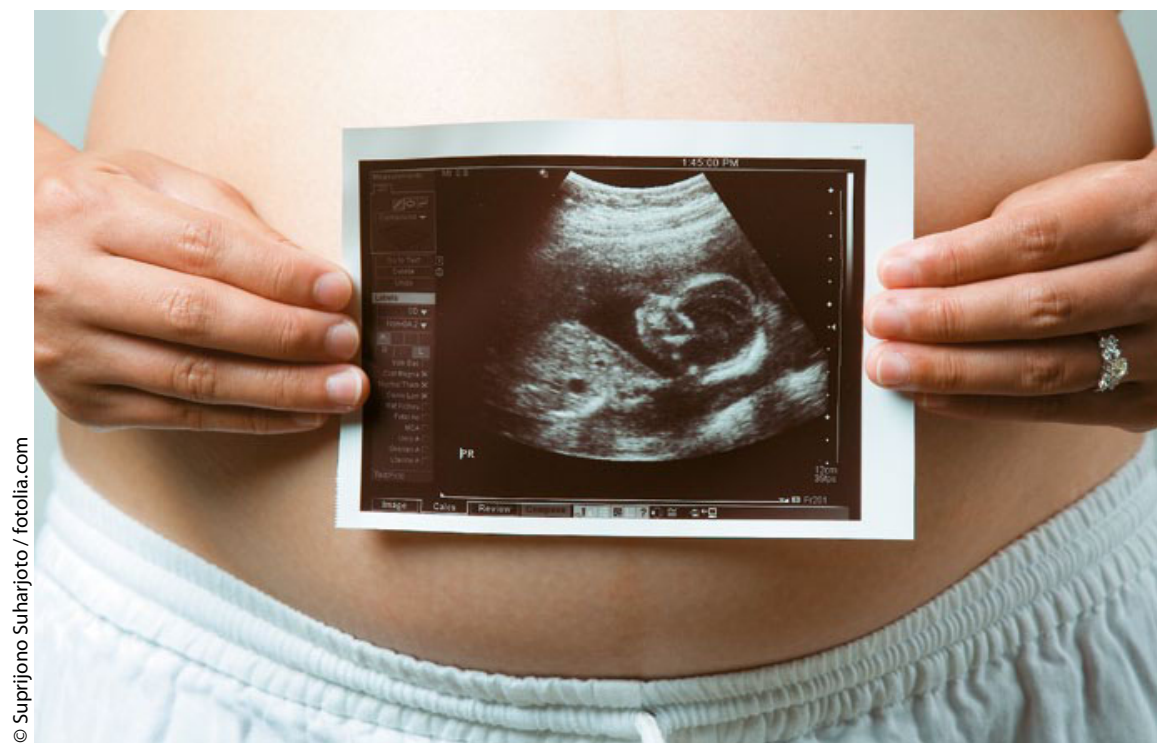

Um das RSV-Risiko für das Kind zu minimieren, sollten die Vitamin-D-Spiegel bei der Mutter im Normbereich bleiben.

$\mathrm{D}$ as RSV ist der wichtigste Erreger von Atemwegsinfektionen bei kleinen Kindern. 10\% dieser Kinder entwickeln ernsthafte tiefe Atemwegsinfektionen. Obwohl einige Risikofaktoren bekannt sind, ist bei der Mehrheit der schweren Erkrankungen ein solcher nicht zu identifizieren. Es gab in der Vergangenheit schon Hinweise darauf, dass ein subklinischer Vitamin-D-Mangel die Wahrscheinlichkeit tiefer Atemwegsinfektionen bei Säuglingen erhöht. Eine Arbeitsgruppe aus Utrecht/Niederlande ist nun in einer prospektiven Studie der Frage nachgegangen, ob ein Vitamin-D-Mangel der Mutter das Risiko schweren Erkrankungen der unteren Atemwege durch RSV bei den Kindern erhöht.

Bei 156 reifen Neugeborenen wurde das 25-Hydroxy-Vitamin-D im Nabelschnurblut bestimmt. Bei 27\% dieser Kinder fanden sich deutlich verminderte Spiegel unter $50 \mathrm{nmol} / \mathrm{l}$. Diese Verminderung war in den Wintermonaten am deutlichsten ausgeprägt. Die betroffenen Kinder zeigten in der Folgezeit ein 6-fach erhöhtes Risiko einer tiefen Atemwegsin- fektionen durch RSV verglichen mit Kindern, die normale Vitamin-D-Spiegel aufwiesen. Alle Infektionen waren virologisch gesichert

Belderbos ME et al. Cord blood vitamin D deficiency is associated with respiratory syncytial virus bronchiolitis. Pediatrics 2011; 127: e1513-20

Kommentar: Zunächst wundert man sich, dass in einem so hoch entwickelten Land wie den Niederlanden ein Mangel an Vitamin D bei Schwangeren so häufig vorkommt. Die Studie zeigt auch, dass Maßnahmen zur Verbesserung des mütterlichen Vitaminstatus das Risiko von RSV-Infektion bei Kindern vermindern könnten. Hier sei auch daran erinnert, dass die Infektanfälligkeit von Säuglingen und Kleinkindern durch Vitamin-D-Mangel erhöht wird. Zwar wird bei Schwangeren allgemein die tägliche Supplementierung mit 400 Einheiten $(10 \mu \mathrm{g})$ Vitamin D empfohlen. Die optimale Dosis ist jedoch unklar. Hier sind weitere Untersuchungen dringend wünschenswert. Dr. Hartmut Koch
Rachenmandeln bei Infekten raus?

Ein Autorenteam aus den Niederlanden untersuchte in einer offenen multizentrischen randomisierten Studie, ob infektgeplagte Kinder nach einer Adenoidektomie tatsächlich seltener krank sind. 111 Kinder zwischen einem und vier Jahren nahmen an der Studie teil, alle mit rezidivierenden Infekten der oberen Atemwege in der Vorgeschichte. Ein Teil der kleinen Patienten wurde operiert, bei den anderen abgewartet. Die Kinder profitierten im Nachbeobachtungszeitraum von 24 Monaten nicht von dem Eingriff. Die Operierten hatten 7,90-mal pro Personenjahr einen Infekt, die Nicht-Operierten 7,84-mal. Auch die Infektdauer unterschied sich nicht: Kinder, denen Rachenmandeln entfernt wurden, waren 66,10 Tage pro Personenjahr krank, die Kinder, bei denen man abwartete, 67,36 Tage. Auch bei Mittelohrentzündungen brachte die Operation keinerlei Vorteil. Während die Operation keinen Einfluss auf das Infektgeschehen hatte, sank mit steigendem Alter ganz allgemein die Zahl der Infektionen. Dr. Dagmar Kraus

van den Aardweg MTA et al. BMJ 2011; 343: d5154

\section{Häufig Myokarditis bei Herztod}

Kanadische Pädiater haben in einer Studie 4.926 Autopsien von Kindern und Jugendlichen aus dem Zeitraum von 1984-2003 retrospektiv analysiert. Dabei stießen sie auf 103 Fälle von plötzlichem unerwartetem Herztod. Die 52 Mädchen und 51 Jungen waren zwischen einem Tag und 15 Jahren alt geworden. 35,9\% der Fälle entpuppten sich auf dem Obduktionstisch als Myokarditis, 18,4\% als Linksherzhypoplasie-Syndrom (HLHS) und 16,5\% als dilatative Kardiomyopathie (DCM). Anomalien der Koronararterien, Aortenstenosen oder Gefäßtranspositionen waren deutlich seltener. Das Durchschnittsalter der Patienten, die an HLHS gestorben waren, lag bei 6,5 Tagen. DCM-Patienten wurden im Schnitt 1,7 und MyokarditisKranke 5,4 Jahre alt. Nur rund jeder Vierte $(26,2 \%)$ hatte vor seinem Tod Warnsymptome verspürt. 40,5\% der Patienten mit Myokarditis entwickelten (meist grippeähnliche) Symptome. Von den DCMPatienten zeigten nur 31,5\% Beschwerden. Bei $10,5 \%$ der HLHS-Patienten waren Probleme beim Füttern aufgefallen.

Dr. Robert Bublak

Ilina MV et al. Pediatrics 2011; 128: e513 